\title{
From Weak to Strong Watermarking
}

\author{
Nicholas Hopper ${ }^{1}$, David Molnar ${ }^{2}$, and David Wagner ${ }^{2}$ \\ ${ }^{1}$ University of Minnesota, Minneapolis MN 55455, USA \\ hopper@cs . umn.edu \\ ${ }^{2}$ University of California, Berkeley, Berkeley CA 94720, USA \\ $\{$ dmolnar, daw $\}$ @eecs. berkeley.edu
}

\begin{abstract}
The informal goal of a watermarking scheme is to "mark" a digital object, such as a picture or video, in such a way that it is difficult for an adversary to remove the mark without destroying the content of the object. Although there has been considerable work proposing and breaking watermarking schemes, there has been little attention given to the formal security goals of such a scheme. In this work, we provide a new complexity-theoretic definition of security for watermarking schemes. We describe some shortcomings of previous attempts at defining watermarking security, and show that security under our definition also implies security under previous definitions. We also propose two weaker security conditions that seem to capture the security goals of practice-oriented work on watermarking and show how schemes satisfying these weaker goals can be strengthened to satisfy our definition.
\end{abstract}

\section{Introduction}

Informally, a digital watermarking scheme is a procedure which embeds a "mark" in an object so that it is hard to remove the mark without "damaging" the object. These procedures have a wide variety of applications to digital rights management, including detection of unauthorized copies, limitations on media copying, tracing of information leaks, and resolution of ownership disputes over digital content; for further exposition on various applications see, for example [1, ch. 20]. As a result, watermarking schemes have seen intense research efforts; for example, see [2] and

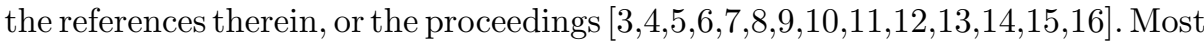
of this work is focused on the construction of schemes for various digital media and attacks on these schemes, where there is a long history of schemes being broken almost immediately after they are proposed.

Given this history, it is not surprising that in the security community, there is a perception that secure watermarking is "theoretically impossible," as expressed, for instance, in 1/17/8. While this idea is intuitively appealing, it is difficult to prove something is (im)possible without first formally defining the notion. Consider for instance, the related notions of program obfuscation and steganography, which were both widely believed to be impossible. Program obfuscation was formalized and shown to be impossible in general [19, but subsequently some progress has been made in limited cases 2021. Steganography, in contrast, was formalized and shown to be possible, but at limited rates [22 2324]. 
Surprisingly, formal definitions for watermarking security have only recently appeared in the literature. The state of the art focuses on defining schemes secure against specific "protocol attacks," which attack the protocols that use a watermark rather than removing a mark from an object [25]; these very powerful attacks changed researchers' understanding of what it means for a watermark to be "secure." For example, Kutter et al. 26] introduced the copy attack, in which a watermark is copied from an object $O_{1}$ into an object $O_{2}$ to form an object $\mathrm{O}_{2}^{\prime}$ that appears marked even though it was never legitimately watermarked. This makes it impossible to use the attacked watermarking scheme for various applications, such as resolving ownership disputes.

Later Adelsbach, Katzenbeisser, and Veith formalized copy attacks and a different protocol attack known as an ambiguity attack. They then showed protocols intended to be provably secure against these attacks [27]. Several other authors have also produced schemes claimed to be provably resistant to copy attacks or other protocol attacks 28,29] 11 While this line of work has led to interesting results, there are some limitations, which we summarize in [30]. Additionally, this approach leads to an "arms race," in which, as new protocol attacks are discovered, new watermarking schemes must be designed and proven secure.

The primary contribution of this work is to initiate the systematic study of watermarking security definitions. We define a "strong watermarking" security condition with respect to a metric space on objects, which compares a watermark to an ideal functionality in which an object is marked if and only if it is similar to some object previously marked by the functionality. We show that this definition implies security against previously known protocol attacks, and explore the question of proving impossibility. We also explore weaker security conditions and show how, under some conditions, schemes satisfying these weaker definitions can be strengthened or amplified to produce strong watermarks.

We stress that in these latter results, we explicitly do not construct "secure" watermarking schemes from scratch. Instead, we show that watermark designers can achieve a strong notion of security from weak constructions that are not secure against protocol attacks. These results have two implications. First, impossibility results for strong watermarking in a metric space will also imply impossibility of these weaker goals. Second, this means that watermark designers need not complicate their schemes by attempting to rule out protocol attacks. Instead, they need only achieve the weaker notion and then apply our results; put another way, it is enough to build schemes that heuristically satisfy these goals and apply our constructions to build (heuristically) strong watermarking schemes, similar to results that say we can build (heuristically) strong secret-key encryption schemes from (heuristically) strong block ciphers.

Overview of our results. In Section 3 we propose a new definition of secure watermarking schemes, that we call strong watermarking, in the case that the marking and detecting procedures share a secret key. Our definition allows the

\footnotetext{
${ }^{1}$ We stress that these constructions, similarly to our own, do not attempt to construct a provably secure watermark "from scratch" but rather try to build something "secure against X" from a watermark that is not assumed to be secure in this sense.
} 
adversary to make adaptive queries to oracles for both marking an object and detecting whether an object is marked. The main idea of the definition is that a strong watermarking scheme (in which there is no communication between the marking and detection procedures) should simulate an "ideal watermarking functionality," which we define. We show that strong watermarking implies security against all known protocol attacks, and argue that the definition will imply security against future protocol attacks. Furthermore, we show that security in our model depends critically on both the notion of similarity and the distribution on objects to be marked; specifically, we show an example of these settings under which strong watermarking is impossible, and an example where strong watermarking exists, relative to an oracle.

In Section 4 we introduce a "weaker" notion of watermark, which we call a non-removable embedding. This is a weak notion because it only requires that the watermark cannot be removed; we explicitly allow copy and ambiguity attacks to succeed against non-removable embeddings. We formalize this notion, prove a separation between the notion and our proposed strong definition, and point out that many watermarking schemes in the literature use a security metric closely related to this notion. We also introduce a notion of "limited" adversaries, who only create new objects based on some limited set of transformations. This notion is interesting since there are some techniques in the watermarking literature which seem to imply provable security against "limited" attacks such as Gaussian noise. Additionally, some applications of watermarking only require watermarks to be "robust" against distortions caused by physical processes; these can be modeled by limited adversaries. We note that all of our results on amplification can be easily extended to the limited adversarial setting. We then show how schemes that are provably secure under the strong watermarking definition can be constructed from non-removable embeddings plus a semi-offline trusted third party, a standard digital signature scheme, and a semantically secure symmetric encryption scheme. This shows that our notion of strong watermarking can be built on the "weak" primitive of non-removable embeddings. While we do require a third party, this party is not required during watermark detection.

In Section 5 we study an alternative method for producing a strong watermarking scheme. Specifically, we consider the question of security amplification of watermarking schemes. We formally specify two new notions that correspond to a weaker version of strong watermarking and show how schemes which satisfy these natural conditions can be efficiently composed to produce strong watermarking schemes. Note that this construction can be seen as an heuristic method to create strong watermarking schemes as well as a way to extend impossibility results for a given notion of similarity.

\section{Preliminaries}

We will work with discrete metric spaces. A discrete metric space $\mathcal{M}$ is a finite space equipped with a distance function $d: \mathcal{M} \times \mathcal{M} \rightarrow \mathbb{Z}^{+} \cup\{0\}$. The distance function is symmetric, obeys the triangle inequality and has the property that if $d(x, y)=0$ then $x=y$. We will associate with a metric space a similarity 
relation $\sim$ defined by $x \sim_{\delta} y \equiv d(x, y) \leq \delta$ for some fixed $\delta$. When the meaning is clear from context, we will drop the $\delta$ and simply write $\sim$. For simplicity, we will assume that all parties can efficiently evaluate $\sim$. Finally, we denote by $\mathcal{D}$ a distribution on $\mathcal{M}$. Unless otherwise specified, we assume that all parties can efficiently sample from $\mathcal{D}$ and we denote by $O \leftarrow_{R} \mathcal{D}$ an object $O \in \mathcal{M}$ sampled according to the distribution $\mathcal{D}$.

We will also make use of a digital signature scheme $\mathcal{S}=\{$ SGen, Sig, Ver $\}$. We say that a signature scheme is $(t, q, \epsilon)$-existentially unforgeable under adaptive chosen message attack 31 if all adversaries running in time at most $t$ making at most $q$ queries to a signature oracle have chance at most $\epsilon$ of obtaining a signature on a message not previously queried.

We will use a symmetric encryption scheme $\mathcal{S E}=\{$ Encrypt, Decrypt $\}$. We say that a symmetric encryption scheme is $(t, q, \epsilon)$-secure in the left-or-right sense [32] if every time $t$ adversary, given $q$ queries to a "left-or-right" oracle $\operatorname{LOR}_{K}\left(b, x_{0}, x_{1}\right)=\operatorname{Encrypt}\left(K, x_{b}\right)$ cannot distinguish between the case that $b=$ 0 and $b=1$ with advantage better than $\epsilon$.

Finally, we will need a pseudorandom function ensemble $\left\{F:\{0,1\}^{k} \times\{0,1\}^{L(k)} \rightarrow\{0,1\}^{\ell(k)}\right\}_{k \in \mathbb{N}}$ 33. We say that a function $F$ is $(t, q, \epsilon)$-pseudorandom if any adversary running in time at most $t$ and making at most $q$ queries to a function oracle can distinguish an oracle for $F\left(U_{k}, \cdot\right)$ from an oracle for a random function $f:\{0,1\}^{L(k)} \rightarrow\{0,1\}^{\ell(k)}$ with advantage at most $\epsilon$.

\section{Strong Watermarking}

As previously mentioned, the informal notion of a watermarking scheme requires the ability to somehow "mark" digital objects, such as pictures, sound, video, or text. The scheme should also satisfy several additional requirements:

- The result, $O^{\prime}$, of marking an object, $O$, should be "similar" to $O$.

- An adversary, given $O^{\prime}$, should not be able to find an object $O^{\prime \prime}$ that is similar to $O^{\prime}$ but unmarked; this prevents removal of the mark except by "damaging" the object.

- Most objects $O$ must not be marked. If this is not the case, then certain desirable uses of watermarks, such as searching for copies of $O^{\prime}$ and proving ownership of $O^{\prime}$, are not possible.

- There should be no communication required between the marking procedure and the detecting procedure; or this communication should be minimized. This is necessary for many applications, for example, a media player that may not have a network connection.

We will model the notion of similarity or damage by postulating the existence of a "perceptual metric" that measures the distance between objects of a given type. Thus such a metric would assign a small distance between two pictures that look alike and a large distance between two very different pictures. In practice it is difficult to characterize such a metric space, so researchers typically focus on Euclidean or weighted $L_{1}$ distance in some "perceptually significant" space such 
as the Fourier [34], Wavelet [35], or Fourier Mellin [36] transforms. Once we fix a metric $d$, the natural notion of similarity is the relation $\sim_{\delta}$ defined previously, that is, we will say that objects $O_{1}$ and $O_{2}$ are similar if $d\left(O_{1}, O_{2}\right) \leq \delta$.

Given this formalization of similarity, we can construct a perfectly secure watermarking scheme that optimally satisfies the above requirements. To mark an object $O$ with key $K$, the ideal scheme simply adds $O$ to its list of objects marked with $K$; to test whether an object $O^{\prime}$ is marked with $K$, the ideal scheme simply searches the appropriate list of marked objects and returns true if it finds an object similar to $O^{\prime}$ and false otherwise. This "ideal" scheme does not allow an adversary to succeed in "unmarking" a marked object but leaves the largest possible set of objects unmarked subject to this constraint. The ideal scheme is undesirable in that it requires unbounded, online communication between the marking and detection algorithms; our intent is to compare a real-world watermarking scheme (which does not allow any online communication between the marking and detection procedures) to this ideal.

An informal statement of our definition allows an adversary access to a marking oracle and a detection oracle for a watermarking scheme. The adversary then attempts to attack the scheme by finding an object such that the results of the actual detection algorithm and the ideal detection procedure differ: either the object is marked and should not be, or it is unmarked and should be. Unfortunately, any watermarking scheme that produces objects that are similar to its input and has a static detection scheme would be insecure under this definition. The intuition is that the following attack would succeed with very high probability:

1. The adversary samples an object $O \in \mathcal{M}$. Since it has not been queried to the marking procedure, it is not yet marked under the ideal scheme.

2. Next the adversary queries $\operatorname{Mark}(O)$, to get an object $O^{\prime}$ similar to $O$.

3. Finally, the adversary queries $\operatorname{Detect}(O)$. In the watermarking scheme under attack, $O$ should not be marked (since it was not marked in step 1 , and there is no communication between marking and detection schemes). But in the ideal scheme, it is close to $O^{\prime}$, which is marked. Thus the adversary has succeeded in finding an object on which the real and ideal schemes differ.

We give a formal proof of this in 30, where we also show that a cryptographically natural alternative definition also rules out secure schemes that distort originals by less than half the similarity radius. Our solution is to introduce a third, challenge oracle that selects objects to watermark from some probability distribution; the performance of the watermarking scheme is only compared to that of the ideal scheme on these challenge objects.

\subsection{Definition of Strong Watermarking Schemes}

A secret-key watermarking scheme $\mathcal{W}=\{$ WMGen, Mark, Detect $\}$ consists of three algorithms: WMGen : $1^{*} \rightarrow$ Keys generates a secret key to be used in marking and detection; Mark : Keys $\times \mathcal{M} \rightarrow \mathcal{M}$ takes a key and an object to 
Oracle $\operatorname{Mark}^{*}(O)$ :

1. $O^{\prime} \leftarrow \operatorname{Mark}(K, O)$

2. Marked $\leftarrow$ Marked $\cup\left\{O^{\prime}\right\}$

3. $\operatorname{return}\left(O^{\prime}\right)$
Oracle $\operatorname{Detect}^{*}(O)$ :

1. $b \leftarrow \operatorname{Detect}(K, O)$

2. $B^{\prime} \leftarrow$ IdealDetect $(O)$

3. if $b \notin B^{\prime}$

4. then bad $\leftarrow$ true

5. $\operatorname{return}(b)$
Oracle Challenge ${ }_{\mathcal{D}}^{*}()$

1. $O \leftarrow_{R} \mathcal{D}$

2. $O^{\prime} \leftarrow \operatorname{Mark}(K, O)$

3. chalns $\leftarrow$ chalns $\cup\left\{O^{\prime}\right\}$

4. Marked $\leftarrow$ Marked $\cup\left\{O^{\prime}\right\}$

5. $\operatorname{return}\left(O^{\prime}\right)$

Fig. 1. Definition of Mark*, Challenge* , and Detect* oracles for strong watermarking. The global variables $K$, Marked, chalns, and bad are initialized in Figure 2.

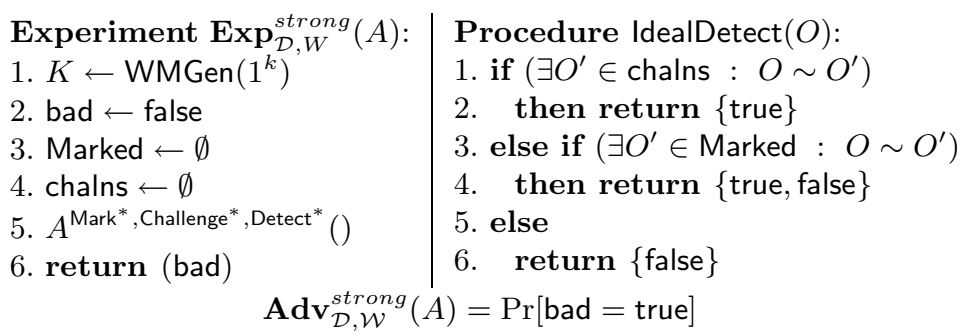

Fig. 2. Definition of security experiment for strong watermarking

mark and returns a new object; and Detect : Keys $\times \mathcal{M} \rightarrow\{$ true, false $\}$. Notice that we do not explicitly allow any online communication between the Detect and Mark procedures, since in many applications the devices detecting and marking objects may not have any means by which to communicate.

We can now define strong watermark security. Our definition formalizes the informal discussion above. An adversary is given access to oracles for Mark and Detect, and a special Challenge* oracle that samples and marks objects from an efficiently sampleable distribution $\mathcal{D}$ over $\mathcal{M}$. The adversary wins if he calls Detect* on an object that is either marked, but not similar to the result of a Mark* or Challenge* query, or unmarked, but similar to the result of some Challenge* query. Notice that unlike in the hypothetical discussion above, we only require the objects near the result of Mark (rather than the input) to be marked, since these are (presumably) the ones that the adversary will be able to access. The formal security experiment has four global variables: Marked and chalns, sets of objects; bad, a boolean flag; and $K$, a key. In Figures 1 and 2 we show pseudocode for initializing the security experiment and the ideal detection functionality, as well as for oracles Mark*, Challenge*, and Detect*. We note that some of our reductions require the ability to sample from the distribution $\mathcal{D}$ on $\mathcal{M}$.

We say that a watermark is $\rho$-preserving for $\mathcal{D}$ if $\operatorname{Pr}\left[K \leftarrow\right.$ WMGen $\left(1^{k}\right) ; O \leftarrow{ }_{R}$ $\mathcal{D} ; O^{\prime} \leftarrow \operatorname{Mark}(K, O): d\left(O, O^{\prime}\right)>\rho$ ] is negligible in $k$; that is, if the marked version of an object is almost always within distance $\rho$ of the original. This "bounded distortion" requirement is not strictly necessary for security in all applications, but is typically vital to the utility of a watermarking scheme.

The advantage of an adversary $A_{\text {Strong }}$ is $\mathbf{A} \mathbf{d v}_{\mathcal{D}, W}^{\text {strong }}\left(A_{\text {Strong }}\right)$ as defined in Figure 2. The scheme is a $\left(\mathcal{D}, t, q_{M}, q_{D}, q_{C}, \epsilon, \delta\right)$-strong watermarking scheme if 
for all adversaries $A_{\text {Strong }}$ running in time at most $t$, making at most $q_{M}$ queries to Mark*, at most $q_{D}$ queries to Detect*, and at most $q_{C}$ queries to Challenge* , the advantage of $A_{\text {Strong }}$ is at most $\epsilon$ with respect to similarity relation $\sim_{\delta}$.

Philosophically, one may think of the above experiment as a game between, say, a "hacker" and a "studio." The hacker can "give" movies to the studio to see how they look when marked, and he can check, using his personal DVD player, whether any particular object is marked. Meanwhile, the studio will release other videos not created by the hacker; it is the hacker's goal to "unmark" one of these movies, or alternatively, to create a movie that appears to be marked but was never marked by the studio. If the hacker cannot do this, the studio can have good confidence that a movie will appear marked iff it was produced by them.

Dependence on $\sim$ and $\mathcal{D}$. It should be clear that the existence of strong watermarks depends critically on both the similarity relation $\sim$ and the distribution on challenge objects, $\mathcal{D}$. For instance, if an attacker can deduce, given the result of a query to Challenge $e_{\mathcal{D}}^{*}$, the object $O \leftarrow_{R} \mathcal{D}$ from line 1 of Figure 1 then as pointed out in our earlier discussion, the scheme cannot be secure for $\mathcal{D}$ and $\sim$. Thus $\mathcal{D}$ must have high entropy, and Mark must be "one-way" for most keys. Likewise, if for any given $O$, enumerating the set $N_{\delta}(O)=\left\{O^{\prime}: O^{\prime} \sim O\right\}$ is feasible, then a watermarking scheme cannot be secure. In this work, we do not explore all the necessary conditions on $\sim$ and $\mathcal{D}$; it seems to be a difficult challenge to even identify the correct similarity metric and distribution for many of the applications of watermarking. Here we briefly give two results that show that even when the previous two conditions are satisfied, there cannot be a "generic" argument for the existence or impossibility of strong watermarks.

Proposition 1. Let $\mathcal{D}$ be the uniform distribution on $k$-bit strings and let $d(x, y)$ be the hamming distance metric on $k$-bit strings. Then there is no $\delta$-preserving, $\left(\mathcal{D}, O(k), 1,1,1,1 / 2^{\delta+1}, \delta\right)$-strong watermarking scheme.

Notice that for $\delta(k)=O(\log k)$, the neighbor set has size superpolynomial in $k$, and $\mathcal{D}$ has $k$ bits of entropy, yet no watermarking scheme can have security better than $1 / 2 k$. The proposition can be seen to be true as follows. Suppose we uniformly pick a point $x \in\{0,1\}^{k}$; consider the point $y$ returned by $\operatorname{Mark}^{*}(x)$, and let $z$ and $w$ be uniformly chosen points in $N_{\delta}(y)$ and $N_{\delta}(x)$, respectively. Now we know that if a watermarking scheme is to be $\varepsilon$-secure, it must be that $\operatorname{Pr}\left[\operatorname{Detect}^{*}(z)=\right.$ false $] \leq \varepsilon$, since otherwise an adversary can remove a mark with probability greater than $\epsilon$ by sampling a random point in the neighborhood of a marked object. It can also be shown that $\operatorname{Pr}\left[z \in N_{\delta}(x)\right] \geq 1 / 2^{\delta}$. This gives us that $\operatorname{Pr}\left[z \in N_{\delta}(x) \wedge \operatorname{Detect}^{*}(z)=\right.$ true $] \geq 1-\left(\operatorname{Pr}\left[z \notin N_{\delta}(x)\right]+\operatorname{Pr}\left[\operatorname{Detect}^{*}(z)=\right.\right.$ false $\left.]\right) \geq$

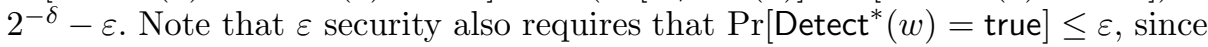
otherwise we can easily find a marked point - by randomly sampling an object in the neighborhood of a random point - breaking the watermark. Thus we also have that $\varepsilon \geq \operatorname{Pr}\left[\operatorname{Detect}^{*}(w)=\right.$ true $\left.\wedge w \in N_{\delta}(y)\right]$. But by symmetry, for any fixed 


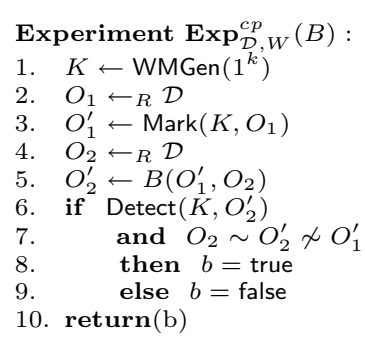

$\mathbf{A d v}_{\mathcal{D}, W}^{c p}(B)=\operatorname{Pr}[b=$ true $]$
Experiment $\operatorname{Exp}_{\mathcal{D}, W}^{a m b}(B)$ :

1. $K \leftarrow$ WMGen

2. repeat

3. $\quad O_{1} \leftarrow_{R} \mathcal{D}$

4. until $\operatorname{Detect}\left(K, O_{1}\right)=$ false

5. $O_{1}^{\prime} \leftarrow B\left(O_{1}\right)$

6. if $\operatorname{Detect}\left(K, O_{1}^{\prime}\right)$ and $O_{1} \sim O_{1}^{\prime}$

7. then $b=$ true

8. else $b=$ false

9. $\operatorname{return}(b)$

$\mathbf{A d v}_{\mathcal{D}, W}^{a m b}(B)=\operatorname{Pr}[b=$ true $]$
Adversary $A_{c p}^{B}()$

1. $O_{1} \leftarrow \operatorname{Mark}^{*}\left(O \leftarrow_{R} \mathcal{D}\right)$

2. $\mathrm{O}_{2} \leftarrow{ }_{R} \mathcal{D}$

3. $\mathrm{O}_{2}^{\prime} \leftarrow B\left(O_{1}^{\prime}, O_{2}\right)$

4. Detect* $\left.{ }^{*} O_{2}^{\prime}\right)$

Adversary $A_{a m b}^{B}$ :

1. $O_{1} \leftarrow R \mathcal{D}$

2. Detect ${ }^{*}\left(O_{1}\right)$

3. $O_{1}^{\prime} \leftarrow B\left(O_{1}\right)$

4. Detect* $^{*}\left(O_{1}^{\prime}\right)$

Fig. 3. Experiments for copy and ambiguity attacks and the corresponding strong watermark adversary

choice of $K, x, y$, we have $\operatorname{Pr}\left[\operatorname{Detect}^{*}(w)=\right.$ true $\left.\wedge w \in N_{\delta}(y)\right]=\operatorname{Pr}\left[\operatorname{Detect}^{*}(z)=\right.$ true $\left.\wedge z \in N_{\delta}(x)\right]$. This gives $\varepsilon \geq 2^{-\delta}-\varepsilon$, or $\varepsilon \geq 2^{-\delta-1}$.

Notice that a similar argument applies to any metric space, distribution and marking function such that (i) the neighborhood of an object and its marked version are symmetric, (ii) these neighborhoods have noticeable intersection, and (iii) it is possible to uniformly sample from the neighborhood set of an object. Thus to rule out an impossibility result, we seek to violate these properties.

Proposition 2. There exists an oracle $\Pi$, relative to which there exists a distribution $\mathcal{D}_{\Pi}$, a metric $d_{\Pi}$, and a 1-preserving watermarking scheme $W^{\Pi}$ such that $W^{\Pi}$ is $\left(\mathcal{D}_{\Pi}, t, t, t, t, t^{2} / 2^{k}, 1\right)$-strong.

Intuitively, we will choose $\Pi, d_{\Pi}$ and $\mathcal{D}_{\Pi}$ so that for most strings $x$ it will be very hard to even find a string $y$ such that $d_{\Pi}(x, y)=1$, but the oracle gives us a way to sample from a set of "special" strings $x^{\prime}$ that violate this property. Once we mark an object $x^{\prime}$ it is no longer in this special set, so it is hard for the adversary to remove the mark. Formally, the oracle $\Pi$ "knows" a uniformly chosen bijection $\pi:\{0,1\}^{2 k} \rightarrow\{0,1\}^{k} \times\{0,1\}^{k}$ for each $k$ and answers three types of queries: sample, dist, and move. $\Pi$ (sample, $y$ ) returns $\pi^{-1}\left(y, 0^{k}\right)$. $\Pi$ (dist, $\left.x_{0}, x_{1}\right)$ computes $\left(y_{b}, z_{b}\right)=\pi\left(x_{b}\right)$, and then returns 0 if $x_{0}=x_{1}, 1$ if $y_{0}=y_{1}$ and some $z_{b}=0^{k}, 2$ if $y_{0}=y_{1}$, and 3 otherwise. $\Pi$ (move, $x, z^{\prime}$ ) computes $(y, z)=\pi(x)$; if $z=0^{k}$ then it returns $\pi^{-1}\left(y, z^{\prime}\right)$; if $z=z^{\prime}$ it returns $\pi^{-1}\left(y, 0^{k}\right)$, and otherwise it returns $x$. The distribution $\mathcal{D}_{\Pi}$ is defined as $\Pi$ (sample, $U_{k}$ ) and the metric $d_{\Pi}(x, y)=\Pi($ dist $, x, y)$, so that for most $2 k$-bit strings $x$, there is only one string at distance 1 from $x$. The marking scheme $W^{\Pi}$ uses $k$-bit keys, and computes Mark ${ }^{\Pi}(K, x)=\Pi($ move, $x, K)$, while Detect $^{\Pi}(K, x)$ returns true iff $\Pi$ (move, $x, K) \neq x$.

We remark that, obviously, the oracle distribution $\Pi$ does not prove that strong watermarks exist. It merely shows that there cannot be a "black-box" proof that rules out all possible strong watermarking schemes without considering the details of $\mathcal{D}$ and $\sim$. We believe it is an interesting open question to find any $\mathcal{D}$ and $\sim$, even if they are contrived, that provably admit a strong watermarking scheme without reference to an oracle, or even with small values $\left(q_{M}, q_{C}, q_{D}\right)$. 


\subsection{Strong Watermarks Are Secure Against Protocol Attacks}

Adelsbach et al. provided the first formal definition of copy attacks and ambiguity attacks [27. We adapt their definitions to our setting, in which we consider only the presence of a mark rather than its content. We show that strong watermarks are secure against copy and ambiguity attacks.

First we consider copy attacks. Informally, a copy attack occurs when an adversary can "copy" a watermark from a marked object $O_{1}^{\prime}$ to a second object $O_{2}$. In our watermarking model, "copy" means that the adversary, given a marked object $O_{1}^{\prime}$, can cause an object $O_{2}$ to return true for Detect* despite never having been queried to Mark. More formally, we say a watermarking scheme is $\left(\mathcal{D}, t, \epsilon_{c p}, \delta_{c p}\right)$-secure against copy attacks if all adversaries $B$ running in time at most $t$ have advantage $\mathbf{A d v}_{\mathcal{D}, \mathcal{W}}^{c p}(B) \leq \epsilon_{c p}$ with respect to similarity relation $\sim \delta_{c p}$. Notice that in this definition (and in the original definition of Adelsbach et al. 27]) the copy adversary is not afforded access to a Mark* or Detect* oracle. We can prove that a $\mathcal{D}$-strong watermarking scheme is not vulnerable to copy attacks for any sampleable distribution $\mathcal{D}^{\prime}$ : if there exists an adversary $B$ that successfully carries out a copy attack, then the adversary $A_{c p}^{B}$ in Figure 3 succeeds at breaking the strong watermark. A formal theorem statement and proof are in 30 .

Next, we consider ambiguity attacks. A classical ambiguity attack takes an unmarked object $O_{1}$, and produces a new "original" object $O_{2}$ such that $O_{1}$ appears to be marked with $\mathrm{O}_{2}$ as the original. In our model, we can recast ambiguity attacks as, given an unmarked object $O_{1}$, find an object $O_{2}$ such that $\mathrm{O}_{2} \sim \mathrm{O}_{1}$ and $\mathrm{O}_{2}$ appears to be marked, without legitimately marking $\mathrm{O}_{2}$. Strong watermarking implies security against ambiguity attacks: if $B$ succeeds at carrying out an ambiguity attack, then the adversary $A_{a m b}^{B}$ shown in Figure 3 breaks the strong watermark. Details are in [30].

Remark. We note that some works on protocol attacks describe attacks where the adversary is allowed to choose the key to the watermarking scheme. While it is important to eventually address such chosen-key attacks, we believe it is an interesting and important first step to concentrate on getting the definitions right for the more basic scenario. Thus in this paper we do not consider attacks that involve manipulating the keys of the marking and detection procedures.

\section{Non-removable Embeddings and Strong Watermarks}

Many watermarking schemes in the literature actually provide a somewhat different interface from the watermarking primitive described in the previous section. Instead, these schemes focus on embedding a short string within an object so that if the adversary does not distort the object too much, the embedded string can be recovered. Typical schemes do not attempt to prevent "insertion" of strings into an object, which is the reason that many protocol attacks succeed. In this section, we give a formal notion of a primitive, the non-removable embedding (NRE), that seems to capture this design goal. We will demonstrate that NREs 


$$
\begin{aligned}
& \begin{array}{l|l}
\text { Experiment } \operatorname{Exp}_{\mathcal{D}}^{N R E}(A): & \text { Oracle Challenge } \\
& *(m) \text { : }
\end{array} \\
& \text { 1. }\left(z, z^{\prime}\right) \leftarrow \operatorname{EMGen}\left(1^{k}\right) \quad \text { 1. } O \leftarrow{ }_{R} \mathcal{D} \\
& \text { 2. Embedded } \leftarrow \emptyset \\
& \text { 3. } O_{A} \leftarrow A^{\operatorname{Embed}(z, \cdot, \cdot), \text { Challenge }}{ }^{*}\left(z^{\prime}\right) \quad 3 \text {. Embedded } \leftarrow \text { Embedded } \cup\left\{\left(O^{\prime}, m\right)\right\} \\
& \text { 4. return } O^{\prime} \\
& \operatorname{Adv}_{\mathcal{D}}^{N R E}(A)=\operatorname{Pr}\left[\exists\left(O_{i}, m_{i}\right) \in \text { Embedded : } O_{A} \sim O_{i} \wedge \operatorname{Extract}\left(z^{\prime}, O_{A}\right) \neq m_{i}\right]
\end{aligned}
$$

Fig. 4. Security experiment and Embed* oracle for non-removable embeddings

are provably weaker objects than strong watermarks: if NREs exist at all, then there are NREs that allow copy attacks. After separating the notions of NREs and strong watermarks, we give a construction which makes limited use of a semitrusted third party to construct a strong watermarking scheme from a NRE.

The notion of an NRE is closely related to a security notion widespread in the watermarking literature. Many schemes presented in the watermarking literature, for example 3738,3940,41, take as their evaluation metric the bit error rate for a watermarked message given a specified constraint on the distortion allowed the adversary, or "watermark to noise ratio." Essentially, these schemes attempt to bound the rate of bit errors in the embedded string for a given amount of distortion induced by the adversary. One of the interesting properties of the NRE notion is that we can easily build an NRE from such schemes. Because we deal with probabilistic polynomial time adversaries, we can assume that the bit errors follow a computationally bounded distribution. Therefore, we can use the coding methods of Micali et al. to obtain an NRE from up to a bit error rate of one half: we simply encode the message before embedding and decode on extraction 42 .

To begin, an embedding scheme (Embed, Extract, EMGen) is a triple of algorithms with the following signatures: Embed : Aux $\times \mathcal{M} \times\{0,1\}^{k} \rightarrow \mathcal{M}$, Extract : Aux ${ }^{\prime} \times \mathcal{M} \rightarrow\{0,1\}^{k} \cup \perp$, and EMGen : $1^{*} \rightarrow$ Aux $\times$ Aux $x^{\prime}$ for some fixed $k$. Here $\mathcal{M}$ is a metric space, and Aux and Aux' are sets of possible auxiliary inputs. For example, Aux might be a set of secret keys, while Aux' might be a set of public keys. $k$ is the length of strings to be embedded in objects.

We further require that embedded messages can be extracted, i.e. for $\left(z, z^{\prime}\right) \leftarrow$ $\operatorname{EMGen}\left(1^{k}\right)$, we have $\operatorname{Extract}\left(z^{\prime}, \operatorname{Embed}(z, O, x)\right)=x$ with high probability. An embedding scheme is $\rho$-preserving for $\mathcal{D}$ if for all $m \in\{0,1\}^{k}, d(\operatorname{Embed}(O, m), O)$ $\leq \rho$ with high probability over $O \leftarrow_{R} \mathcal{D}$. Together, these give a correctness and a bounded distortion requirement for a non-removable embedding.

We define security of embedding scheme NRE by saying it is $\left(\mathcal{D}, t, q_{E}, q_{C}, \epsilon, \delta\right)$ non-removable for distribution $\mathcal{D}$ if for all $A$ running in time at most $t$, that make at most $q_{E}$ queries to an Embed oracle and at most $q_{C}$ queries to the Challenge* oracle, the advantage $\mathbf{A d v}_{\mathcal{D}}^{N R E}(A)$ defined in Figure 4 is at most $\epsilon$.

Remarks. This definition does not rule out the protocol attacks we have discussed: in particular, if there is a $\rho$-preserving non-removable embedding for the metric space $\mathcal{M}$ with metric $d$, we can construct a $2 \rho$-preserving non-removable 
embedding for the metric space $\mathcal{M} \times\{0,1\}^{k}$ with metric $d^{\prime}$, that allows copy attacks to succeed, as follows. We define the metric $d^{\prime}\left(\left(O_{1}, y_{1}\right),\left(O_{2}, y_{2}\right)\right)$ to be $d\left(O_{1}, O_{2}\right)$ if $y_{1}=y_{2}$ and $d\left(O_{1}, O_{2}\right)+\rho$ otherwise; define Embed $(z,(O, y), x)=$ $(\operatorname{Embed}(z, O, x), x)$, and $\operatorname{Extract}{ }^{\prime}\left(z^{\prime},(O, x)\right)=\operatorname{Extract}\left(z^{\prime}, O\right)$ if $\operatorname{Extract}\left(z^{\prime}, O\right) \neq \perp$ and Extract ${ }^{\prime}\left(z^{\prime},(O, x)\right)=x$ otherwise. Then it is easy to see that, as long as $\rho<\delta$, given a marked object $O=\left(O_{1}, x\right)$ and an unmarked object $O^{\prime}=\left(O_{2}, y\right)$ we can "copy" the mark from $O$ onto $O^{\prime}$ by setting $O^{\prime \prime}=\left(O_{2}, x\right)$; yet it is still hard to remove $x$ from $O$.

Although we do not explicitly require it, we note that typical applications will require that $\rho<\delta$ and in many cases, $\rho \ll \delta$. We also note that it is trivial to construct a $\rho$-preserving non-removable embedding for the case that $\rho=\sup _{(x, y) \in \mathcal{M} \times \mathcal{M}} d(x, y)$, using an error correcting code with minimum distance $2 \delta$, if one exists for the metric space $\mathcal{M} 2$ Thus the interesting question, for a given metric space, becomes "for what values of $(\rho, \delta)$ is a NRE possible?"

Barak et al. 43 defined watermarking for circuits, showing there are families of circuits for which such watermarking is impossible, and that the notion is incompatible with obfuscation even for watermarks that only succeed on some circuits. They briefly discuss how allowing "approximate implementations" may change their results. Our definition, in contrast, places these decisions in the choice of $\sim$ and the distribution $\mathcal{D}$.

We also note that many "public-key" watermarking schemes in the literature seem to target $\left(\mathcal{D}, t, q_{E}, 1, \epsilon, \delta\right)$ non-removability, expressed in terms of bit error rate for the watermarked message as noted above. A simple hybrid argument implies such schemes also have $\left(\mathcal{D}, t, q_{E}, q_{C}, q_{C} \epsilon, \delta\right)$ non-removability 4441]. Thus while we are not aware of any strong candidate NREs, the existence of such a scheme seems to be a natural assumption if watermarking can be feasible at all.

We note that Moulin and Wang have shown that quantization index modulation (QIM) techniques provide provably good watermarks against an adversarial memoryless channel. The restriction to memoryless channels, together with an assumption that the host signal is Gaussian, allows them to analytically derive the "worst possible" channel and evaluate the bit error rate for a watermark signal under a specified bound on the mean squared error introduced by the adversary. Therefore, we can view their result as showing that QIM techniques yield a non-removable embedding for the class of memoryless adversary channels. While this is a severely limited class of adversaries, it shows that our notion is realizable at least under "toy" circumstances.

Finally, the StirMark benchmark 4546 performs transformations such as resampling, resizing, and "jitter" in images; this benchmark is widely used to evaluate watermarks. We can capture both Moulin and Wang's result and the StirMark benchmark in our framework. If $\mathcal{C}$ is a set of object transformations, we define an attacker from class $\mathcal{C}$ to be an adversary who can only create objects via

\footnotetext{
${ }^{2}$ We let $\operatorname{Embed}(O, x)=\operatorname{encode}(x)$ and $\operatorname{Extract}(O)=\operatorname{decode}(O)$. If the code's minimum distance is $2 \delta$ then clearly any distortion by distance $\delta$ or less will result in extraction of the "embedded" message, but the worst-case distortion of this procedure is the maximum possible distance between two objects in $\mathcal{M}$.
} 
sampling from $\mathcal{D}$, queries to oracles, and applying transformations from $\mathcal{C}$ to objects he has already created. Then it is a straightforward extension of our results to show that if there is an NRE that is secure against all attackers from class $\mathcal{C}$, there is a strong watermarking scheme that is secure against all attackers from $\mathcal{C}$.

\subsection{Building Strong Watermarks from Embeddings}

We now show how to build ideal watermarking schemes from non-removable embeddings, digital signature schemes, and a trusted third party (TTP). The main benefit of our scheme is that the TTP need not be present during watermark detection; anyone can check whether an object is marked without needing to contact the TTP in a wide variety of cases. Our scheme requires digital signatures in addition to a TTP because the underlying embeddings are not assumed secure against insertion of watermarks or copy attacks. The nonremovable embedding is necessary to allow offline detection, because otherwise an adversary could remove any metadata that might be attached to an object as a mark.

The TTP has well-known public keys and provides two services over secure channels: $\operatorname{Register}(O, K, x)$ picks a unique identifier $i$, checks that $x=$ $\operatorname{Encrypt}(K, O)$, and returns $\left(i, \operatorname{Sig}_{T T P}(i, x)\right)$; Retrieve $(i)$ returns the $x$ associated with $i$ if any exists, or $\perp$ otherwise; we assume that neither call returns until a correctly authenticated response is received. We require that parties who execute Mark can communicate with the TTP as necessary. However, Retrieve is implemented in a semi-offline manner. Unique identifiers are assigned in ascending order, and the TTP publishes a signed list, TTPList, of all $(i, x)$ pairs each day. Consequently, Retrieve( $i$; TTPList) only needs to contact the TTP if $i>$ TTPList.length. Standard measures (such as substituting a zero-knowledge proof of knowledge of $(O, K)$ for $(O, K)$; maintaining an ordered, signed TTPList; checking for consistency of TTP lists between updates; et cetera) can be taken to reduce the level of trust required in the TTP; we omit them for clarity of presentation, and because they do not affect the security proof.

Now let $\mathcal{E}=$ (Embed, Extract, EMGen) be an embedding; and let $\mathcal{S E}=$ (Encrypt, Decrypt) be a symmetric encryption scheme. We then define a new watermarking scheme $\mathcal{W}_{\mathcal{E}}=\left(\mathrm{WMGen}_{\mathcal{E}, \mathcal{S E}}\right.$, Mark $_{\mathcal{E}, \mathcal{S E}}$, Detect $\left._{\mathcal{E}, \mathcal{S E}}\right)$ as shown in Figure 5. Mark $(O)$ encrypts $O$, registers the ciphertext with the TTP, and embeds the TTP's identifier and signature in $O$. Detect $(O$; TTPList) extracts the TTP identifier and signature, retrieves the associated ciphertext, and checks that $O$ is close to the result of Embed applied to the plaintext.

The main result of this section is that if the underlying embedding is nonremovable, then the scheme $\mathcal{W}_{\mathcal{E}}$ satisfies our notion of strong watermarking. Formally, we can state the following theorem, whose proof is in [30].

Theorem 1. Suppose $\mathcal{E}$ is a $\left(\mathcal{D}, t_{E}, q_{E M}, q_{E C}, \epsilon_{E}, \delta\right)$-secure non-removable embedding, $S=$ (SGen, Sig, Ver) is $\left(t_{S}, q_{S}, \epsilon_{S}\right)$-existentially unforgeable under chosen message attack, and $\mathcal{S E}=($ Encrypt, Decrypt $)$ is $\left(t, q_{e n}, \epsilon_{e n}\right)$ left-or-right secure under chosen plaintext attack. Then $\mathcal{W}_{\mathcal{E}}$ is a $\left(t^{\prime}, q_{M}, q_{D}, q_{C}, \epsilon^{\prime}, \delta\right)$-strong watermarking scheme, where $\epsilon^{\prime}=2 \epsilon_{S}+\epsilon_{\text {en }}+\epsilon_{E}, q_{M}+q_{C} \leq \min \left(q_{e n}, q_{S}\right)$, $q_{M} \leq q_{E M}$, and $q_{C} \leq q_{E C}$. 

Algorithm $\operatorname{Mark}_{\mathcal{E}}\left(\left(z, z^{\prime}, K\right), O\right)$
1. $x \leftarrow \operatorname{Encrypt}(K, O)$
2. $(i, \sigma) \leftarrow \operatorname{Register}(O, K, x)$
3. $O^{\prime} \leftarrow \operatorname{Embed}(z, O,(i, \sigma))$
4. return $O^{\prime}$

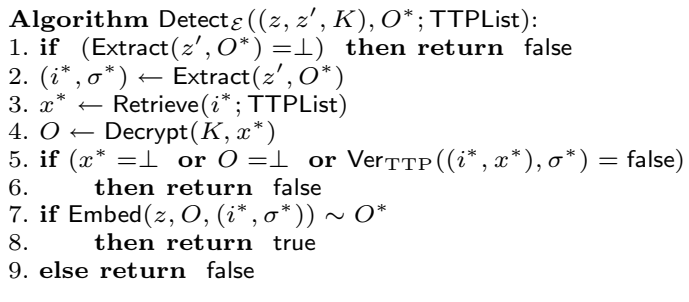

Algorithm WMGen ${ }_{\mathcal{E}}\left(1^{k}\right)$

1. $\left(z, z^{\prime}\right) \leftarrow \operatorname{EMGen}\left(1^{k}\right)$

2. $K \leftarrow_{R}\{0,1\}^{k}$

3. return $\left(z, z^{\prime}, K\right)$

Fig. 5. Pseudocode for $\mathrm{WMGen}_{\mathcal{E}}, \mathrm{Mark}_{\mathcal{E}}$, and Detect $_{\mathcal{E}}$

Remarks. We note that the scheme as written requires the Embed procedure to be deterministic; this is without loss of generality because the shared symmetric key between Mark and Detect can include a seed for a pseudorandom function that is used to generate the random bits used by Embed in a deterministic way from its arguments, without changing the security properties of the scheme.

We also note that if the distribution $\mathcal{D}$ has Shannon entropy less than $k$ - the length of strings embedded by $\mathcal{E}$ - then in principle the TTP can be removed from this scheme. In this case, the marking scheme first losslessly compresses the object $O$ into a short string $x$ of length less than $k$, and the string $x$ is then encrypted and authenticated using standard cryptographic techniques to get a ciphertext $c$ which is embedded into $O$. The detection scheme recovers $c$, checks it for authenticity and if it passes, decrypts $c$ to obtain $x$, then expands $x$ to the original object $O$ before comparing it to the input object. Thus our TTP can be seen as implementing a compression algorithm for unknown or incompressible distributions $\mathcal{D}$.

\section{Strengthening Watermarks by Composition}

Suppose we are given a watermarking scheme with known attacks that succeed at insertion or removal of a watermark with high probability, for example $90 \%$, but retains some weak sense of security, in that it is not known how to defeat it with probability 1. In this section, we show that this sense of security is essentially enough for strong watermarking. Given an offline watermarking scheme $W$ that satisfies two weak properties, we can construct an (offline) strong watermarking scheme in the sense of Section 3. The first property is that the scheme is secure in this weak sense - every adversary fails to defeat the scheme with some constant probability. The second property is that marking an object many times preserves some similarity to the original.

As mentioned previously, we believe this results has both positive and negative applications. Many of the heuristic watermarking schemes in the literature are broken, but frequently the known attacks do not succeed with probability 1 . Thus applying our amplification scheme could heuristically create schemes which are, in some sense, secure "against known attacks." On the other hand, our results show that in order to rule out even weakly secure watermarking schemes for a given metric and distribution, it is sufficient to concentrate on showing the impossibility of a strong watermarking scheme. 


\subsection{Weakly Secure Watermarking Schemes}

Our scheme will work by applying the Mark function to its own output several times. Because our security notions depend on the probability distribution on the inputs to Mark, we will need some assumption on the distribution of the outputs of Mark. The strongest assumption is that these distributions are identical, but in general this amounts to assuming that Mark is the identity function. Thus, instead, we assume that the (weak) security of a watermark holds even if we make some small distortions to an object before marking it. Formally, we say that a randomized algorithm $D$ is a $(t, r)$-perturbation of $\mathcal{D}$ if $D$ runs in time $t$ and $\operatorname{Pr}\left[O \leftarrow_{R} \mathcal{D} ; O^{\prime} \leftarrow D(O): d_{\mathcal{M}}\left(O, O^{\prime}\right)>r\right]$ is negligible. We will say that our watermarking schemes are weakly secure for $\mathcal{D}$ if they are weakly secure for any $(t, r)$-perturbation of $\mathcal{D}$.

(WEAK) SECURITY AGAINST REMOVAL. We define the removal advantage of an adversary against a watermarking scheme to be the probability that an adversary can produce, given a watermarked object drawn from a $(t, r)$-perturbation of $\mathcal{D}$, a similar object that is not marked. Formally, define

$$
\begin{array}{r}
\operatorname{Adv}_{W, D}^{r m}(\mathcal{A})=\operatorname{Pr}\left[K \leftarrow W \cdot W M G e n\left(1^{k}\right) ; O \leftarrow{ }_{R} \mathcal{D} ; O^{\prime} \leftarrow W \cdot \operatorname{Mark}_{K}(D(O)) ;\right. \\
\left.O^{\prime \prime} \leftarrow \mathcal{A}\left(O^{\prime}\right): W \cdot W_{\text {Detect }}\left(O^{\prime \prime}\right)=\text { false } \wedge O^{\prime \prime} \sim_{\delta} O^{\prime}\right] .
\end{array}
$$

Then, we say that a watermark $W$ is $\left(t, \epsilon_{r m}, \delta, \mathcal{D}, r\right)$-secure against removal if for every time- $t$ adversary $\mathcal{A}$, and every $(t, r)$-perturbation $D$ of $\mathcal{D}, \mathbf{A d v}_{W, D}^{r m}(\mathcal{A}) \leq$ $\epsilon_{r m}$. Informally, this definition says that every adversary who runs in time at most $t$ fails to remove the watermark of an object drawn from a $(t, r)$ perturbation of $\mathcal{D}$ with probability at least $1-\epsilon_{r m}$.

We remark that this experiment captures the intuitive notion of trying to remove a watermark without damaging some challenge object, a common goal of attacks on watermarking schemes found in the literature. We also note that the goal of our scheme is to strengthen a watermark with only constant security against removal - meaning that we explicitly allow a watermarking scheme that can be removed, say, $99 \%$ of the time.

(Weak) Security against insertion. We informally define the insertion advantage of an adversary against a watermarking scheme to be the probability that an adversary can produce, given a single watermarked object, another watermarked object. Formally, define

$$
\begin{aligned}
\operatorname{Adv}_{W, D}^{i n s}(\mathcal{A})=\operatorname{Pr}[ & K \leftarrow \mathrm{WMGen}\left(1^{k}\right) ; O \leftarrow \mathcal{A}\left(1^{k}\right) ; O^{\prime} \leftarrow W \cdot \operatorname{Mark}_{K}(O) ; \\
O^{\prime \prime} & \left.\leftarrow \mathcal{A}\left(O^{\prime}\right): \quad W \cdot \operatorname{Detect}_{K}\left(O^{\prime \prime}\right)=\operatorname{true} \wedge O^{\prime \prime} \chi_{\delta} O^{\prime}\right] .
\end{aligned}
$$

Then, we say that a watermark $W$ is $\left(t, \epsilon_{\text {ins }}, \delta\right)$-secure against insertion if for every time- $t$ adversary $\mathcal{A}, \mathbf{A d v}_{W, D}^{\text {ins }}(\mathcal{A}) \leq \epsilon_{\text {ins }}$. Informally, this definition says that every adversary who runs in time $t$ must fail to produce a (new) watermarked object with probability at least $1-\epsilon_{\text {ins }}$. We remark that security against insertion is essentially an adversarial notion of the "false positive rate" of a watermark 227. 
We can now state the main result of this section; the proof depends on several additional results proved in the remainder of the section:

Theorem 2. Suppose there exists a watermarking scheme $W$ such that:

- $W$ is $\rho$-preserving;

- $W$ is both $\left(t, \epsilon_{r m}, \delta, \mathcal{D}, k^{O(1)} \rho\right)$-secure against removal and $\left(t, \epsilon_{\text {ins }}, \delta\right)$-secure against insertion; and

$-\epsilon_{r m}, \epsilon_{\text {ins }}$ are constants such that $4 \epsilon_{\text {ins }} \lg \frac{1}{\epsilon_{r m}}<1$; and $t=k^{\omega(1)}$

Then there exists a $\left(\mathcal{D}, t^{\prime}, q_{M}, 1, q_{D}, \nu, \delta\right)$-strong watermarking scheme $W^{\prime}$, where $t^{\prime}=k^{\omega(1)}$ and $\nu=1 / k^{\omega(1)}$. The scheme $W^{\prime}$ is $k^{O(1)} \rho$-preserving.

Proof. The new watermark $W^{\prime}$ is constructed from $W$ using the techniques developed in the remainder of this section: first the "alternating" composition $\mathrm{ALT}_{\ell}$ with $\ell=O(\lg k)$ levels, from Section 5.3 is applied to $W$. By repeated application of Theorem 3 the resulting scheme $\mathrm{S}(W)$ is $\nu$-secure against removal and insertion, for negligible $\nu$. Lemma 1 implies that this scheme is also a $\left(\mathcal{D}, t^{\prime}, q_{M}, 1, q_{D}, \nu, \delta\right)$-strong watermark, for $q_{M}+q_{D}=1$. To achieve arbitrary $q_{M}$ and $q_{D}$, we construct the scheme $S^{\prime}(W)$ described in Section 5.4 with $m=q_{M}+q_{D}$. By Theorem 4 the resulting scheme is a $\left(\mathcal{D}, t^{\prime}, q_{M}, 1, q_{D}, \nu, \delta\right)$ strong watermark.

\subsection{Single-Property Amplification}

Let $\mathbb{K}=\left(K_{1}, K_{2}, \ldots, K_{m}\right)$ be a set of independently chosen secret keys. We define $\operatorname{Mark}_{\mathbb{K}}^{W}(O):=W \cdot \operatorname{Mark}_{K_{m}}\left(W \cdot \operatorname{Mark}_{K_{m-1}}\left(\ldots W \cdot \operatorname{Mark}_{K_{1}}(O) \ldots\right)\right)$, i.e. Mark $\mathbf{K}_{\mathbb{K}}^{W}$ is the sequential marking of an object $O$ with each secret key in the vector $\mathbb{K}$. We now have two choices for defining the $\operatorname{Detect}_{\mathbb{K}}^{W}\left(O^{\prime}\right)$ algorithm, each resulting in a different watermarking scheme. Define the schemes as follows:

$$
\begin{aligned}
\operatorname{AND}(m, W) \cdot \operatorname{Detect}_{\mathbb{K}}\left(O^{\prime}\right) & =\bigwedge_{1 \leq i \leq m} W \cdot \operatorname{Detect}_{K_{i}}\left(O^{\prime}\right) \\
\operatorname{OR}(m, W) \cdot \operatorname{Detect}_{\mathbb{K}}\left(O^{\prime}\right) & =\bigvee_{1 \leq i \leq m} W \cdot \operatorname{Detect}_{K_{i}}\left(O^{\prime}\right)
\end{aligned}
$$

Intuitively, we expect that $\operatorname{AND}(m, W)$ will improve the insertion security of watermark $W$ while impeding the removal security. This is because to insert a watermark one must insert $m$ copies of $W$, while to delete a watermark one need only delete 1 out of $m$. Likewise, we intuitively would expect that $\operatorname{OR}(m, W)$ will decrease the insertion security while increasing the removal security. We can write this formally in the following theorem, whose proof is in 30].

Theorem 3. Let $W$ be $\rho$-preserving, $\left(t, \epsilon_{\text {ins }}, \delta\right)$-secure against insertion, and $\left(t, \epsilon_{r m}, \delta, \mathcal{D}, r\right)$-secure against removal. Then:

(a) $\operatorname{OR}(m, W)$ is $\left(t^{\prime}, m \epsilon_{i n s}, \delta-m \rho\right)$ secure against insertion.

(b) $\operatorname{AND}(m, W)$ is $\left(t^{\prime}, m \epsilon_{r m}, \delta-m \rho, \mathcal{D}, r-m \rho\right)$ secure against removal. 
Where $t^{\prime}=t-m T_{M}-O(1)$ if $T_{M}$ is the time to mark an object. Furthermore, for any $q(k) \in k^{O(1)}$,

(c) $\operatorname{AND}(m, W)$ is $\left(t^{\prime}, \epsilon_{i n s}^{m}+1 / q, \delta-m \rho\right)$ secure against insertion.

(d) $\operatorname{OR}(m, W)$ is $\left(t^{\prime}, \epsilon_{r m}^{m}+1 / q, \delta-m \rho, \mathcal{D}, r-m \rho\right)$ secure against removal.

Where $t^{\prime}=t / \operatorname{poly}(q, m)$.

\subsection{Simultaneous Amplification}

Let $W$ be a watermarking scheme with key space $K$ and define the scheme $\operatorname{ALT}(W)$ with key space $K^{4}$ by $\operatorname{ALT}(W)=\operatorname{AND}(2, \operatorname{OR}(2, W))$. Then by the previous theorem, if $W$ is $\left(k^{\omega(1)}, c / 2, \delta, \mathcal{D}, r\right)$ secure against removal and $\left(k^{\omega(1)}, d / 4, \delta\right)$ secure against insertion, then $\operatorname{ALT}(W)$ is $\left(k^{\omega(1)}, c^{2} / 2, \delta-4 \rho, \mathcal{D}, r-4 \rho\right)$-secure against removal and $\left(k^{\omega(1)}, d^{2} / 4, \delta-4 \rho\right)$-secure against insertion. If we define the

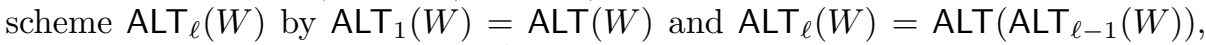
we see that $\operatorname{ALT}_{\ell}(W)$ is $\left(k^{\omega(1)}, d^{2^{\ell}} / 4, \delta-4^{\ell} \rho\right)$-secure against insertion and $\left(k^{\omega(1)}\right.$, $\left.c^{2^{\ell}} / 2, \delta-4^{\ell} \rho, \mathcal{D}, r-4^{\ell} \rho\right)$-secure against removal, for $\ell=O(\log k)$. By setting $\ell=\lceil\log k\rceil$ and letting $\mathrm{S}(W)=\mathrm{OR}\left(2, \operatorname{ALT}_{\ell}(W)\right)$ we obtain a scheme that inserts $\operatorname{poly}(k)$ marks such that any poly $(k)$-time adversary has negligible advantage for both removal and insertion, if the original scheme is weakly secure against (for example) subexponential time adversaries.

Intuitively, we can think of this scheme as building a tree of marking schemes over the object $O$ to be marked. By building the tree appropriately, alternating AND and OR at each level, we can reduce both the insertion and deletion probabilities for the resulting detection scheme. Each leaf of the tree corresponds to an independently keyed insertion of a watermark. Suppose we have a depth $t$ tree comprising $2^{t}$ independent keys. The top gate, an OR, will recursively compute AND.Detect $\left(O, k[1] \ldots k\left[2^{t-1}\right]\right)$ and AND.Detect $\left(O, k\left[2^{t-1}\right] \ldots k\left[2^{t}\right]\right)$ and return true if at least one recursive branch returns true. OR is defined analogously. Alternatively, from the bottom-up view, there is one object in which we may have embedded $n=2^{t}$ marks; we check if each mark is present and then compute a formula based on these truth values to decide whether the composed mark is present.

We note that the full alternating binary tree only exponentially reduces the insertion and removal probabilities if we start with $\epsilon_{r m}<1 / 2$ and $\epsilon_{\text {ins }}<1 / 4$. For many watermarking schemes in the literature, however, we might expect that the insertion probability is low, say $\epsilon_{\text {ins }}<1 / 100$, while the removal probability is high, say $\epsilon_{r m}=0.9$. In this case, we can make the lowest level of the tree consist of an OR of 20 marks to get $\epsilon_{r m}^{\prime}=1 / e^{2}<1 / 2$ and $\epsilon_{\text {ins }}^{\prime}<1 / 5$. We can then build a binary tree on top of the resulting watermark.

It remains to show that the scheme $\mathbf{S}(W)$ is correct, i.e. that S.Detect $_{\mathbb{K}}$ $\left(\mathrm{S}^{M_{a r k}} \mathbb{K}_{\mathbb{K}}(\mathcal{D})\right)=$ true except with negligible probability. Notice, however, that S.Detect returns true if either its left branch or its right branch return true. But the insertion of the marks in the right branch is just one particular instance of an adversary (against the left branch) that returns an output that is 
distorted by distance at most $4^{\ell} \rho$ from its input, so if $\delta>4^{\ell} \rho$, the probability that this "adversary" succeeds in removing the mark inserted by the left branch is negligible.

\subsection{Strong Watermark Security from Insertion and Removal Security}

Notice that the definition of $\left(t, \epsilon_{i n s}, \delta\right)$ security against insertion implies $(\mathcal{D}$, $t, 1,1,0, \epsilon_{\text {ins }}, \delta$ )-strong watermark security: any strong watermark adversary $\mathcal{A}$ who makes one Mark* query and one Detect* query can be converted into a weak insertion adversary $\mathcal{B}: \mathcal{B}\left(1^{k}\right)$ simply runs $\mathcal{A}$ until $\mathcal{A}$ makes a query to Mark*, say $O$, and outputs $O ; \mathcal{B}\left(O^{\prime}\right)$ returns $O^{\prime}$ to $\mathcal{A}$ and outputs the object $O^{\prime \prime}$ that $\mathcal{A}$ queries to Detect*. Since the list chalns is empty, submitting an unmarked $O^{\prime \prime}$ will give $b=$ false and $b^{\prime}=$ false, so $\mathcal{A}$ can only win by "inserting" a watermark. Additionally satisfying $\left(t, \epsilon_{r m}, \delta, \mathcal{D}, r\right)$-security against removal implies $(D(\mathcal{D}), t, 0,1,1, \epsilon, \delta)$ strong watermark security for any $D$ that perturbs $\mathcal{D}$ by at most $r$, because an adversary who makes only a single query $O^{\prime} \leftarrow$ Challenge* $(D(\mathcal{D}))$ can only win by querying Detect ${ }^{*}\left(O^{\prime \prime}\right)$ such that:

$-O^{\prime \prime} \sim O^{\prime}$ and $\operatorname{Detect}_{K}\left(O^{\prime \prime}\right)=$ false; if this happens with probability greater than $\epsilon_{r m}$ then the removal security of the scheme is contradicted.

- $d\left(O^{\prime \prime}, O^{\prime}\right)>\delta$ and $\operatorname{Detect}_{K}\left(O^{\prime \prime}\right)=$ true; if this happens with probability greater than $\epsilon_{\text {ins }}$ then the insertion security is violated: an insertion adversary can always draw his challenge object $O^{\prime} \leftarrow D(\mathcal{D})$.

This observation leads to the following lemma:

Lemma 1. If $W$ is $\left(t, \epsilon_{\text {ins }}, \delta\right)$-secure against insertion and $\left(t, \epsilon_{r m}, \delta, \mathcal{D}, r\right)$-secure against removal then $W$ is a $\left(D(\mathcal{D}), t, q_{M}, 1, q_{C}, \epsilon_{\text {ins }}+\epsilon_{r m}, \delta\right)$-strong watermarking scheme, for any distortion function $D \in \operatorname{TIME}(t)$ that perturbs $\mathcal{D}$ by distance at most $r$, and any $q_{C} \leq 1-q_{M}$.

Suppose that we extend the definition of a strong watermark to allow Mark to maintain a local state. Then we can generically increase the number of (mark and challenge) queries we are secure against by a factor of $n$ while also increasing the running time of Detect by a factor of $n$ as follows. We require that Mark ${ }_{K}^{\prime}$ keeps a count, $i$, of the number of objects it has marked (say modulo $n$ ). When $\operatorname{Mark}_{K}^{\prime}(O)$ marks a new object, it computes the entire set of keys to use as $\mathbb{K}_{i}=F_{K}(i)$, where $F$ is a pseudorandom function of the appropriate output size, and then calls $\operatorname{Mark}_{\mathbb{K}_{i}}(O)$. Then in $\operatorname{Detect}_{K}^{\prime}(O)$ we try $\mathbb{K}=F_{K}(1), F_{K}(2) \ldots F_{K}(n)$ and output true if any of these watermarks is detected. This increases the insertion probability by at most a factor of $n$. We make this more formal in the following theorem, whose proof is in 30.

Theorem 4. Let $W=$ (Mark, Detect) be a (D, $\left.t, q_{M}, 1,1-q_{M}, \epsilon_{w m}, \delta\right)$-strong watermarking scheme and let $W^{\prime}=\left(\right.$ Mark' $^{\prime}$, Detect $\left.{ }^{\prime}\right)$ be a watermarking scheme with the stateful Mark' algorithm described above, and let $F$ be a $\left(t, n, \epsilon_{p r f}\right)$ pseudorandom function. Then $W^{\prime}$ is a $\left(\mathcal{D}, t, q_{M}, 1, n-q_{M}, n \epsilon_{w m}+\epsilon_{p r f}, \delta\right)$-strong watermarking scheme. 


\section{Conclusions}

In this paper we have initiated the scientific study of complexity-based security of watermarking schemes. We define a notion of watermarking security based on comparison to an ideal scheme, and give evidence that this is the right notion of security for watermarks in two ways. First, we show that security in our sense implies previous definitions of security, while the converse is not true. Second, we have shown how to construct a watermark which is secure in our sense from several weaker primitives, which seem to capture the goals of research in watermarking primitives. Our intent is not to introduce new watermarking protocols, but to suggest that security in the "strong watermark" sense is the "right definition": if secure watermarks (in any sense) are feasible at all, then so are strong watermarking schemes. A key question left open by our work, therefore, is the construction of similarity-preserving strong watermarking schemes that are provably-secure under standard cryptographic assumptions; even a construction for a contrived metric space would be an interesting first step in this direction.

\section{Acknowledgments}

We thank Andre Adelsbach, Luis von Ahn, Hayley Iben, Pierre Moulin, and Ahmad-Reza Sadeghi for helpful discussions. We also thank Kannan Ramchandran, Dan Schonberg, and the Berkeley Information Hiding Group for feedback on early versions of this work. Nicholas Hopper was supported by the National Science Foundation, under grant CNS-0546162. David Molnar was supported by a graduate research fellowship from the National Science Foundation and by NSF CCF-0325311. David Wagner was supported by NSF CCF-0325311.

\section{References}

1. Anderson, R.J.: Security Engineering: A Guide to Building Dependable Distributed Systems. John Wiley \& Sons, Inc., New York, NY, USA (2001)

2. Cox, I., Miller, M.L., Bloom, J.A.: Digital watermarking. Morgan Kaufmann Publishers Inc., San Francisco, CA, USA (2002)

3. Wong, P.W., Delp, E.J., eds.: Security and Watermarking of Multimedia Contents, Proceedings. In Wong, P.W., Delp, E.J., eds.: Security and Watermarking of Multimedia Contents. Volume 3657 of Proceedings of SPIE., SPIE (1999)

4. Wong, P.W., Delp, E.J., eds.: Security and Watermarking of Multimedia Contents II, 2000, Proceedings. In Wong, P.W., Delp, E.J., eds.: Security and Watermarking of Multimedia Contents. Volume 3971 of Proceedings of SPIE., SPIE (2000)

5. Wong, P.W., Delp, E.J., eds.: Security and Watermarking of Multimedia Contents III, 2001, Proceedings. In Wong, P.W., Delp, E.J., eds.: Security and Watermarking of Multimedia Contents. Volume 4314 of Proceedings of SPIE., SPIE (2001)

6. Delp, E.J., Wong, P.W., eds.: Security and Watermarking of Multimedia Contents IV, 2002, Proceedings. In Delp, E.J., Wong, P.W., eds.: Security and Watermarking of Multimedia Contents. Volume 4675 of Proceedings of SPIE., SPIE (2002) 
7. Delp, E.J., Wong, P.W., eds.: Security, Steganography, and Watermarking of Multimedia Contents V, 2003, Proceedings. In Delp, E.J., Wong, P.W., eds.: Security and Watermarking of Multimedia Contents. Volume 5020 of Proceedings of SPIE., SPIE (2003)

8. Delp, E.J., Wong, P.W., eds.: Security, Steganography, and Watermarking of Multimedia Contents VI, San Jose, California, USA, January 18-22, 2004, Proceedings. In Delp, E.J., Wong, P.W., eds.: Security, Steganography, and Watermarking of Multimedia Contents. Volume 5306 of Proceedings of SPIE., SPIE (2004)

9. Delp, E.J., Wong, P.W., eds.: Security, Steganography, and Watermarking of Multimedia Contents VII, San Jose, California, USA, January 17-20, 2005, Proceedings. In Delp, E.J., Wong, P.W., eds.: Security, Steganography, and Watermarking of Multimedia Contents. Volume 5681 of Proceedings of SPIE., SPIE (2005)

10. Anderson, R.J., ed.: Information Hiding, First International Workshop, Cambridge, U.K., May 30 - June 1, 1996, Proceedings. In Anderson, R.J., ed.: Information Hiding. Volume 1174 of Lecture Notes in Computer Science., Springer (1996)

11. Aucsmith, D., ed.: Information Hiding, Second International Workshop, Portland, Oregon, USA, April 14-17, 1998, Proceedings. In Aucsmith, D., ed.: Information Hiding. Volume 1525 of Lecture Notes in Computer Science., Springer (1998)

12. Barni, M., Herrera-Joancomartí, J., Katzenbeisser, S., Pérez-González, F., eds.: Information Hiding, 7th International Workshop, IH 2005, Barcelona, Spain, June 6-8, 2005, Revised Selected Papers. In Barni, M., Herrera-Joancomartí, J., Katzenbeisser, S., Pérez-González, F., eds.: Information Hiding. Volume 3727 of Lecture Notes in Computer Science., Springer (2005)

13. Pfitzmann, A., ed.: Information Hiding, Third International Workshop, IH'99, Dresden, Germany, September 29 - October 1, 1999, Proceedings. In Pfitzmann, A., ed.: Information Hiding. Volume 1768 of Lecture Notes in Computer Science., Springer (2000)

14. Moskowitz, I.S., ed.: Information Hiding, 4th International Workshop, IHW 2001, Pittsburgh, PA, USA, April 25-27, 2001, Proceedings. In Moskowitz, I.S., ed.: Information Hiding. Volume 2137 of Lecture Notes in Computer Science., Springer (2001)

15. Petitcolas, F.A.P., ed.: Information Hiding, 5th International Workshop, IH 2002, Noordwijkerhout, The Netherlands, October 7-9, 2002, Revised Papers. In Petitcolas, F.A.P., ed.: Information Hiding. Volume 2578 of Lecture Notes in Computer Science., Springer (2003)

16. Fridrich, J.J., ed.: Information Hiding, 6th International Workshop, IH 2004, Toronto, Canada, May 23-25, 2004, Revised Selected Papers. In Fridrich, J.J., ed.: Information Hiding. Volume 3200 of Lecture Notes in Computer Science., Springer (2004)

17. Gollmann, D.: Computer security. John Wiley \& Sons, Inc., New York, NY, USA (1999)

18. Wikipedia: Digital watermarking — wikipedia, the free encyclopedia (2006) [Online; accessed 31-July-2006].

19. Barak, B., Goldreich, O., Impagliazzo, R., Rudich, S., Sahai, A., Vadhan, S.P., Yang, K.: On the (im)possibility of obfuscating programs. In Kilian, J., ed.: CRYPTO. Volume 2139 of Lecture Notes in Computer Science., Springer (2001) $1-18$

20. Lynn, B., Prabhakaran, M., Sahai, A.: Positive results and techniques for obfuscation. In Cachin, C., Camenisch, J., eds.: EUROCRYPT. Volume 3027 of Lecture Notes in Computer Science., Springer (2004) 20-39 
21. Wee, H.: On obfuscating point functions. In Gabow, H.N., Fagin, R., eds.: STOC, ACM (2005) 523-532

22. Cachin, C.: An information-theoretic model for steganography. In Aucsmith, D., ed.: Information Hiding. Volume 1525 of Lecture Notes in Computer Science., Springer (1998) 306-318

23. Hopper, N.J., Langford, J., von Ahn, L.: Provably secure steganography. In Yung, M., ed.: CRYPTO. Volume 2442 of Lecture Notes in Computer Science., Springer (2002) 77-92

24. Dedic, N., Itkis, G., Reyzin, L., Russell, S.: Upper and lower bounds on black-box steganography. In Kilian, J., ed.: TCC. Volume 3378 of Lecture Notes in Computer Science., Springer (2005) 227-244

25. Craver, S., Memon, N., Yeo, B.L., Yeung, M.M.: Resolving rightful ownerships with invisible watermarking techniques: Limitations, attacks, and implications. IEEE J. SAC 16(4) (May 1998) 573-586 Special issue on copyright \& privacy protection.

26. Kutter, M., Voloshynovskiy, S., Herrigel, A.: The watermark copy attack. In: Proceedings of the SPIE vol. 3971, Security and Watermarking of Multimedia Contents II. (2000) 371-380

27. Adelsbach, A., Katzenbeisser, S., Veith, H.: Watermarking schemes provably secure against copy and ambiguity attacks. In: DRM '03: Proceedings of the 2003 ACM workshop on Digital rights management, ACM Press (2003) 111-119

28. Dittmann, J., Katzenbeisser, S., Schallhart, C., Veith, H.: Provably secure authentication of digital media through invertible watermarks. Cryptology ePrint Archive, Report 2004/293 (2004) http://eprint.iacr.org/

29. Li, Q., Chang, E.C.: On the possibility of non-invertible watermarking schemes. In: Information Hiding Workshop, Springer-Verlag LNCS 3200. (2004)

30. Hopper, N., Molnar, D., Wagner, D.: From weak to strong watermarking. IACR Cryptology ePrint Archive, Report 2006/XXX (2006) http://eprint.iacr.org/2006/XXX

31. Goldwasser, S., Micali, S., Rivest, R.L.: A digital signature scheme secure against adaptive chosen-message attacks. SIAM J. Comput. 17(2) (1988) 281-308

32. Bellare, M., Desai, A., Jokipii, E., Rogaway, P.: A concrete security treatment of symmetric encryption. In: FOCS '97: Proceedings of the 38th Annual Symposium on Foundations of Computer Science (FOCS '97), Washington, DC, USA, IEEE Computer Society (1997) 394

33. Goldreich, O., Goldwasser, S., Micali, S.: How to construct random functions. J. ACM 33(4) (1986) 792-807

34. Li, L., Pan, Z., Zhang, M., Ye, K.: Watermarking subdivision surfaces based on addition property of fourier transform. In: GRAPHITE '04: Proceedings of the 2nd international conference on Computer graphics and interactive techniques in Australasia and South East Asia, New York, NY, USA, ACM Press (2004) 46-49

35. Meerwald, P., Uhl, A.: A survey of wavelet-domain watermarking algorithms. In Wong, P.W., Delp, E.J., eds.: Proceedings of SPIE, Electronic Imaging, Security and Watermarking of Multimedia Contents III. Volume 4314., San Jose, CA, USA, SPIE (January 2001)

36. Ruanaidh, J.J.O., Pereira, S.: A secure robust digital image watermark. In: International Symposium on Advanced Imaging and Network Technologies - Conference on Electronic Imaging: Processing, Printing, and Publishing in Colour. (1998)

37. Moulin, P., Wang, Y.: Improved QIM strategies for gaussian watermarking. In: International Workshop on Digital Watermarking (IWDW '05). (2005) 
38. Bas, P.: A quantization watermarking technique robust to linear and non-linear valumetric distortions using a fractal set of floating quantifiers. In: Information Hiding Workshop (IHW) '05. (2005)

39. Martin, V., Chabert, M., Lacaze, B.: A spread spectrum watermarking scheme based on periodic clock changes for digital images. In: Information Hiding Workshop (IHW) '05. (2005)

40. Doerr, G., Dugelay, J.L.: A quantization watermarking technique robust to linear and non-linear valumetric distortions using a fractal set of floating quantifiers. In: Information Hiding Workshop (IHW) '05. (2005)

41. Hartung, F., Girod, B.: Fast public-key watermarking of compressed video. In: International Conference on Image Processing (ICIP'97). Volume I., Santa Barbara, California, U.S.A. (1997) 528-531

42. Micali, S., Peikert, C., Sudan, M., Wilson, D.: Optimal error correction against computationally bounded noise. In: Theory of Cryptography Conference (TCC) '05. (2005) http://theory.lcs.mit.edu/ ${ }^{\sim}$ cpeikert/pubs/mpsw.ps

43. Barak, B., Goldreich, O., Impagliazzo, R., Rudich, S., Sahai, A., Vadhan, S., Yang, K.: On the (im)possibility of obfuscating programs. In: CRYPTO. (2001)

44. Wong, P.W., Memon, N.: Secret and public key image watermarking schemes for image authentication and ownership verification. IEEE Trans. Image Processing 10(10) (October 2001) 1593-1601

45. Petitcolas, F.A., Anderson, R.J., Kuhn, M.G.: Attacks on copyright marking systems. In: Information Hiding Workshop (IHW) 1998, Springer-Verlag Lecture Notes in Computer Science 1525. (1998) 219-239

46. Petitcolas, F.A.: Watermarking schemes evaluation. IEEE Signal Processing 17(4) (September 2000) 58-64 